\title{
On the structure of the set solutions of a class of paratingent equation with delayed argument
}

\author{
Lotfi Boudjenah \\ Department of Computer Science, University of Oran, Bp 1524, Oran 31000, Algeria
}

Received: 2 October 2015, Revised: 4 March 2016, Accepted: 12 March 2016

Published online: 20 April 2016.

\begin{abstract}
In this paper we will study the main properties of the set solutions of the paratingent equation (type differential inclusion) with delayed argument of the form: $(P t x)(t) \subset F\left([x]_{t}\right)$ for $t \geq 0$ with the initial condition: $x(t)=\zeta(t)$ for $t \leq 0$. We will be interested particularly in the topological properties of emission and zone of emission.
\end{abstract}

Keywords: Attainable set, convex, delayed argument, differential inclusion, emission, paratingent, set solutions.

\section{Introduction}

It is our purpose to study a topological structure of the solution set to the paratingent equation with delayed argument. In [3] we have established the existence of solutions to the paratingent equation with delayed argument of the form: $(P t x)(t) \subset F\left([x]_{t}\right)$ for $t \geq 0$ with the initial condition: $x(t)=\zeta(t)$ for $t \leq 0$, under weak assumptions. In [4] we have established some properties of the set solution, this paper is a continuation of [4]. In this work, we investigate the fundamental properties of emission and zone of emission of the above paratingent equation under the same weak assumptions than in [3]. The study of these properties is justified by the fact that the main topic considered while dealing with differential inclusions is the topological properties of the set solutions. This set, which is consisted of the union of the graphs of all trajectories of the differential inclusions, is called zone of emission or reachable or attainable set of differential inclusions. Wasewski [30], has pointed out that the main properties of trajectories and set solutions of control system are equivalent to the properties of trajectories and set solutions of a certain differential inclusions . Many proofs of existence in optimal control theory depend upon showing that the set solutions is compact. The fact that the set solutions is non-compact causes great difficulties. Wasewski in [28, 29] attempted to overcome this problem by extending the notion of a solution. He introduced the concept of quasitrajectories which are limits of approximate solutions or solutions of a relaxed differential inclusion. This concept is very important in the control theory and its applications. For example, it is well-known that the limit functions of some sequence of trajectories with "bang bang" control are quasitrajectories. Such functions are well-known in automatics as "sliding regimes". The existence of sliding regimes can be established as a result of Turowicz [27] or as a solution to a certain relaxed differential inclusion, Sontag [24, 25], using Filippov theorems, Fronkowska [9].

The first works on paratingent and contingent differential inclusions with delay were published by Myshkis [19]. Moreover, the existence of solution and the main properties of set solution of paratingent and contingent differential inclusions with delay have been studied by a number of authors, for instance, Campu [5,6], Deimling [8], Haddad [10, 11, 12, 13], Kamenskii et all. [15], Kryzowa [17, 18] and Zygmunt [31, 32]. Recent results for differential inclusions with a finite delay in spaces of Banach were obtained by Syam [26] and Castaing-Ibrahim [7]. Recently, Raczynski has 
successfully applied differential inclusions to simulation and modelling theory [20, 21, 22].

For more details on differential inclusions see the books of Aubin and Cellina [1], K. Deimling [8], M. Kamenskii [15] , G. V. Smirnov [23], and M. Kisielewicz [16].

\section{Preliminaries}

Let $(E, \rho)$ and $\left(E^{\prime}, \rho^{\prime}\right)$ two metric spaces. By CompE, we denotes the set of all the nonempty and compact subsets of $E$. If, moreover, $E$ is a vector space, then $C o n v E$ denotes the set of all the convex elements of CompE.

A set-valued map, $F: E \rightarrow C o m p E^{\prime}$, is called upper semi-continuous in $E$, and denoted by u.s.c, if for any point $a \in E$ and all $\varepsilon>0$, there exists $\delta>0$ such that $: x \in B(a)_{\delta} \Rightarrow F(x) \in B(F(a))_{\varepsilon} \quad$ when $B(a)_{\delta}=B(a, \delta)=\{x \in E / \rho(a, x)<\delta\}$ and $B(F(a))_{\varepsilon}=B(F(a), \varepsilon)=\left\{y \in E^{\prime}\right.$ such as $z \in F(a)$ and $\left.\rho^{\prime}(y, z)<\varepsilon\right\}$. (see $[2])$.

We have, on the upper semi-continuity of a set-valued map, the following lemma (see [14]).

Lemma 1. let $(E, \rho)$ and $\left(E^{\prime}, \rho^{\prime}\right)$ two metric spaces. A set-valued map, $F: E \rightarrow C o m p E^{\prime}$, is u.s.c if and only if, for all sequences $\left\{x_{i}\right\} \in E$ and $\left\{y_{i}\right\} \in E^{\prime}$ such that $\left\{x_{i}\right\} \rightarrow x_{0}$ and $\left\{y_{i}\right\} \in F\left(x_{i}\right)$, there exists a subsequence $\left\{y_{i_{k}}\right\}$ of $\left\{y_{i}\right\}$ which is convergent to $y_{0} \in F\left(x_{0}\right)$.

Let $C$ the space of all continuous functions $x: R \rightarrow R^{n}$ with the topology defined by an almost uniform convergence (i.e. a uniform convergence on each compact interval of $R$ ). It is well know that the almost uniform convergence in $C$ is equivalent to the convergence by the metric $\rho$ defined as follows.

$$
\rho(x, y)=\sum_{i=1}^{\infty} \frac{1}{2^{i}} \min \{(1, \sup |x(t)-y(t)|),-i \leq t \leq i\} \text { for } x, y \in C .
$$

then $C$ is a metric locally convex linear topological space. Let $\beta<0$ be a fixed real number and let $I=[0, \infty[\subset R$. If $x \in C$, the symbol $[x]_{t}$ will be denote the restriction of $x$ on the interval $[\beta, t]$ when $t \in I$ and $\|x\|_{t}=\max \{|x(s)|, \beta \leq s \leq t\}$ with $|x|=\max \left\{\left|x_{1}\right|,\left|x_{2}\right|, \ldots,\left|x_{n}\right|\right\}$ for $x=\left(x_{1}, x_{2}, \ldots, x_{n}\right) \in R^{n}$.

Let $G$ denote the metric space whose elements are functions $[x]_{t},[y]_{u}, \ldots$, where $t \in I, u \in I$, the distance between two functions $[x]_{t},[y]_{u}$, being understood as a distance of their graphs in $R \times R^{n}$ in the Hausdorff sense.

\subsection{Paratingent of a function}

Having a function $x \in C$ and $t \in I$, the set of limit points

$$
\lim \frac{x\left(u_{i}\right)-x\left(s_{i}\right)}{u_{i}-s_{i}}=\alpha
$$

where $u_{i} \in I, s_{i} \in I, u_{i} \neq s_{i}(i=1,2, \ldots)$, and $\lim u_{i}=\lim s_{i}=t$, is called the paratingent of $x$ at the point $t$ and denoted by $(P t x)(t)$. It is easy to see that Ptx maps the interval $I$ into the family of the nonempty and closed subsets of $R^{n}$. 


\subsection{Paratingent equation with a delayed argument}

Let a set-valued map $F: G \rightarrow C$ ompR $R^{n}$, a relation of the form:

$$
(P t x)(t) \subset F\left([x]_{t}\right) \text { where } t \in I \text { and } x \in C .
$$

is called paratingent equation with a delayed argument where $t \in I$ and $x \in C$. Every function $x \in C$ satisfying (1) will be called the solution of the equation (1).

The generalized problem of Cauchy for the equation (1) consists in the search for a solution of the equation (1) which will satisfy the initial condition:

$$
x(t)=\xi(t) \text { for } t \in[\beta, 0]
$$

where the function $\xi \in C$, called the initial function is given in advance (i.e. the solution of the equation (1) must contain a certain curve given in advance).

\subsection{Existence solutions}

Assume the following hypothesis.

(H1): The set-valued mapping $F: G \rightarrow C_{\text {Conv }}^{n}$ is upper semi-continuous and satisfies the condition. $F\left([x]_{t}\right) \subset \bar{B}\left(0, w\left(t, \|\left. x\right|_{t}\right)\right)$, for $t \geq 0$ where $\bar{B}(0, r)$ denote the closed ball of center 0 of $R^{n}$ and radius $r, w(t, y)$ is a continuous function from $I \times I$ to $I$, increasing in $y$ and such that the ordinary differential equation: $y \prime=w(t, y)$, with the initial condition $y(0)=A$ ( $A$ an arbitrary real positive number) has a maximal solution, denoted $M(t)$, on all intervals $I$ and for all $A$.

Let $D$ a compact subset of $C$ and $\Phi$ the class of all the functions $x \in C$ satisfying the inequality $|x(t)| \leq M(T)$ for $t \in[0, T], T$ a real positive number arbitrarily fixed. In [3] we have established the following theorem:

Theorem 1. Under the hypothesis (H1), in the class $\Phi$ and for any $\xi \in D$, there is at least a solution of the problem (1)-(2).

From proof of the theorem, it follows.

Corollary 1. If $y \in \Phi$ and $($ Pt $y)(t) \subset \bar{B}\left(0, w\left(t,|| x||_{t}\right)\right)$,we have: $\left|y(t)-y\left(t^{\prime}\right)\right| \leq \Omega_{T}\left|t-t^{\prime}\right|$ for $t, t^{\prime} \in[0, T]$, where $\Omega_{T}=$ $\max \{w(s, M(T)): s \in[0, T]\}$.

\section{Properties of the set solutions}

Definition 1. A function $x$ is called a trajectory of $F\left([y]_{t}\right)$, if $($ Pt $x)(t) \subset F\left([x]_{t}\right)$ for $t \geq 0$. We call emission of the function $\zeta$, the set of all trajectories $x$ of $F\left([y]_{t}\right)$ such that $x \in \Phi$ and $x(t)=\zeta(t)$ for all $t \in[\beta, 0]$. We denote this set by $E_{\Phi}(F, \zeta)$. And, we call emission of the set $D$, the union of emissions of all functions $\zeta \in D$, it is denoted by $E_{\Phi}(F, D)$, i.e. $E_{\Phi}(F, D)=\left\{\cup E_{\Phi}(F, \zeta) / \zeta \in D\right\}$.

We call zone of emission of function $\zeta \in D$, and we denote by $e_{\Phi}(F, \zeta)$, the union of the graphs of the functions belonging to $E_{\Phi}(F, \zeta)$, i.e. : $e_{\Phi}(F, \zeta)=\left\{(t, y) \in R x R^{n} / t \in[\beta, T], y=\zeta(t)\right.$ and $\left.y \in E_{\Phi}(F, \zeta)\right\}$. We call zone of emission of the set $D$, and we denote by $e_{\Phi}(F, D)$, the union of all the zones of emissions of functions $\zeta \in D$, i.e.e $e_{\Phi}(F, D)=\left\{e_{\Phi}(F, \zeta) / \zeta \in D\right\}$. 
Let $\Gamma$ a fixed real number. We denote by $e_{\Phi}(F, \zeta, \Gamma)$ the section of the zone of emission of the function $\zeta$ by the plan $t=\Gamma$, i.e. : $e_{\Phi}(F, \zeta, \Gamma)=e_{\Phi}(F, \zeta) \cap\left\{(t, x) \in R x R^{n} / t=\Gamma\right.$ and $\left.x \in R^{n}\right\}$ and by $e_{\Phi}(F, D, \Gamma)$ the union of sections $e_{\Phi}(F, \zeta, \Gamma)$ where $\zeta \in D$, i.e. $e_{\Phi}(F, D, \Gamma)=\left\{\cup e_{\Phi}(F, \zeta, \Gamma) / \zeta \in D\right\}$.

We recall the following theorems from [4].

Theorem 2. [4] If the application $F: G \rightarrow C o n v R^{n}$ verifies the assumption $H 1$, then the emission $E_{\Phi}(F, \zeta)$ of the function $\zeta$ is a nonempty and compact set of $C$.

Proof. In view of theorem 1, it following that the set $E_{\Phi}(F, \zeta)$ is nonempty. Let $x \in E_{\Phi}(F, \zeta)$ then we have: $\|x\|_{t} \leq M(T)$ for $t \in[0, T], x(t)=\zeta(t)$ for $t \in[\beta, 0]$ and $\left|x(t)-x\left(t^{\prime}\right)\right| \leq \Omega_{T}\left|t-t^{\prime}\right|$ for $t, t^{\prime} \in[0, T]$, where $\Omega_{T}=\max \{w(s, M(T)): s \in$ $[0, T]\}$. The functions belonging to $E_{\Phi}(F, \zeta)$ are uniformly bonded and equicontinuous. Therefore, $E_{\Phi}(F, \zeta)$ is a compact subset of space $C$, according to the theorem of Arzela.

Theorem 3. [4] Consider the set-valued application $E: D \rightarrow C o m p C$ defined by: $E(\zeta)=E_{\Phi}(F, \zeta)$ where $\zeta \in D$. If the application $F$ verifies the assumption $H 1$, then the application $E$ is upper semi-continuous in $D$.

Proof. Let $\left\{\zeta_{i}\right\}, i=1,2, \ldots$, a sequence of functions of $D$ convergent to a function $\zeta \in D$ and $\left\{x_{i}\right\}, i=1,2, \ldots$, a sequence of functions of $\Phi$ such that $x_{i} \in E\left(\zeta_{i}\right)$ for $i=1,2, \ldots$

We have $x_{i} \in E_{\Phi}\left(F, \zeta_{i}\right)$ from where $:\left|x_{i}(t)\right| \leq M(T)$ for $t \in[0, T]$ and $x_{i}(t)=\zeta_{i}(t)$ for $t \in[\beta, 0]$. From corollary 1 we have : $\left|x_{i}(t)-x_{i}\left(t^{\prime}\right)\right| \leq \Omega_{T}\left|t-t^{\prime}\right|$ for $t, t^{\prime} \in[0, T]$, where $\Omega_{T}=\max \{w(s, M(T)): s \in[0, T]\}$. By theorem $2 E_{\Phi}(F, \zeta)$ is compact, then we can extract a subsequence $\left\{x_{i j}\right\}$ of $\left\{x_{i}\right\}$ which converges to a certain function $x$. It is obvious that the function $x$ satisfies the following conditions: $|x(t)| \leq M(T)$ for $t \in[0, T]$ and $x(t)=\zeta(t)$ for $t \in[\beta, 0]$. Otherwise we have : $\left(\right.$ Pt $\left.x_{i j}\right)(t) \subset F\left(\left[x_{i}\right]_{t}\right)$ for $t \geq 0$, it follows, according to lemma 3.7 in [3] (lemma 4 in [31]) that:

$($ Pt $x)(t) \subset F\left([x]_{t}\right)$, for $t \geq 0$, and thus $x \in E(\zeta)$. Finally, we show the upper semi-continuity of the application $E$ by using lemma 1 .

Theorem 4. [4] The emission $E_{\Phi}(F, \zeta)$ of the set $D$ is an nonempty and compact subset of $C$.

Proof. By definition: $E_{\Phi}(F, D)=\{\cup E(F, \zeta) / \zeta \in D\} . E_{\Phi}(F, D)$ is a nonempty set according to the theorem 2 . Let $E: D \rightarrow \operatorname{CompC}$ the application defined in the theorem 3: we have: $E(D)=E_{\Phi}(F, D)$. The application $E$ is upper semicontinuous, in view of theorem 3. $D$ being compact by assumption, therefore $E(D)$ is a compact set.

\subsection{Main results}

Now we prove the results about the section of the zone of emission of the function $\zeta$ by the plan $t=\Gamma$.

Theorem 5. Let $\Gamma$ a fixed positive real number, then the section $e_{\Phi}(F, \zeta, \Gamma)$ is a nonempty and compact subset of RxR ${ }^{n}$.

Proof. Let $\left\{t_{i}, x_{i}\right\}, i=1,2, \ldots$, a sequence of elements of $e_{\Phi}(F, \zeta, \Gamma)$. By definition of $e_{\Phi}(F, \zeta, \Gamma)$, there exist functixons $y_{i} \in E_{\Phi}(F, \zeta)$ such as; $x_{i}\left(t_{i}\right)=y_{i}, i=1,2, \ldots$. The set $E_{\Phi}(F, \zeta)$ is compact, then there exists a subsequence of $\left\{y_{i}\right\}$ which converges to a certain function $y_{0} \in E_{\Phi}(F, \zeta)$. The sequence $\left\{t_{i}\right\}, i=1,2, \ldots$, is bonded, we can extract a subsequence from $\left\{t_{i}\right\}$ which converges to some $t_{0} \in[0, T]$. Let $\{i j\}$ a sequence of indices such as $t_{i j} \rightarrow t_{0}$ and $y_{i j} \rightarrow y_{0}$. We have: $\left(t_{i j}, y_{i j}\right)=$ $\left(t_{i j}, x_{i j}\left(t_{i j}\right)\right) \rightarrow\left(t_{0}, x_{0}\left(t_{0}\right)\right)$ with $\left(t_{0}, x_{0}\left(t_{0}\right)\right) \in e_{\Phi}(F, \zeta, \Gamma)$, from where we have the compactness of the set $e_{\Phi}(F, \zeta, \Gamma)$ in $R \times R^{n}$

Theorem 6. Let $\Gamma$ a fixed positive real number, then the application: $e_{\Gamma}: D \rightarrow \operatorname{CompR}^{n+1}$ defined by $: e_{\Gamma}(\zeta)=e_{\Phi}(F, \zeta, \Gamma)$ where $\zeta \in D$, is upper semi-continuous in $D$. 
Proof. Let $\left\{\zeta_{i}\right\}, i=1,2, \ldots$, a sequence of elements of $D$ such as; $\zeta_{i} \rightarrow \zeta \in D$ and $\left(t_{i}, y_{i}\right) \in e_{\Gamma}\left(\zeta_{i}\right), i=1,2, \ldots$ The set $E_{\Phi}(F, D)$ is compact, there exists a sequence of functions $\left\{x_{i}\right\}$ of $E_{\Phi}(F, D)$ which converges to a certain function $x \in$ $E_{\Phi}(F, D)$. The sequence $\left\{t_{i}\right\}$ is bounded, there exists a subsequence of $\left\{t_{i}\right\}$ which converges to a certain $t \in[0, T]$. Let $\{i j\}$ a sequence of indices such that: $\left\{t_{i j}\right\} \rightarrow t$ and $\left\{x_{i j}\right\} \rightarrow x$. then $\left(t_{i j}, y_{i j}\right)=\left(t_{i j}, x_{i j}\left(t_{i j}\right)\right) \rightarrow(t, x(t))$ and at the same time we have: $\left(\operatorname{Ptx}_{i j}\right)(t) \subset F\left(\left[x_{i j}\right]_{t}\right)$ for $t \in[0, T]$, and $x_{i j}(t)=\zeta_{i j}$ for $t \in[\beta, 0]$. Obviously we have $x(t)=\zeta(t)$ for $t$ $\leq 0$ and according to the lemma 3.7 in [3] (lemma 4 in [31]) we have: $(P t x)(t) \subset F\left([x]_{t}\right)$ for $t \geq 0$, which shows that $x \in E_{\Phi}(F, D)$ and consequently $(t, y)=(t, x(t)) \in e_{\Phi}(F, \zeta, \Gamma)=e_{\Gamma}(\zeta)$. Finally, according to the lemma 1 , we have the upper semi-continuity of the application $e_{\Gamma}$ in $D$.

Theorem 7. Let $\Gamma$ a fixed positive real number, then the set $e_{\Phi}(F, D, \Gamma)$ is a nonempty and compact subset of $R^{n+1}$.

Proof. The set $e_{\Phi}(F, D, \Gamma)=\left\{\cup e_{\Phi}(F, \zeta, \Gamma) / \zeta \in D\right\}=e_{\Gamma}(D)$ where $e_{\Gamma}: D \rightarrow \operatorname{CompR}^{n+1}$ is the application defined in theorem 6. According to theorem 5, the set $e_{\Phi}(F, \zeta, \Gamma)$ is nonempty, therefore $e_{\Phi}(F, D, \Gamma)$ is nonempty. In view of theorem 6, the application $e_{\Gamma}: D \rightarrow \operatorname{CompR}^{n+1}$ is upper semi-continuous and the set $D$ is compact by assumption, then we have compactness of the set $e_{\Gamma}(D)$.

Acknowledgement. The authors would like to thank an anonymous referee for his/her helpful suggestions for improving the original manuscript.

\section{References}

[1] J. P. Aubin, A. Cellina; Differential inclusions, Springer-Verlag, 1984.

[2] C. Berge; Espaces topologiques, fonctions set-valueds, Dunod, Paris, 1966.

[3] L. Boudjenah; Existence of the solutions of the paratingent equation with delayed argument. Electron. J. Diff. Eqns., Vol. 2005, No.14, 1-8, 2005

[4] L Boudjenah; On the properties of the set solutions of a class of paratingent equation with delay. British Journal of Mathematics \& Computer Science 4 (14): 1999-2003, 2014.

[5] E. Campu; Equations différentielles au paratingent à retardement, dans les espaces de Banach. Théoreme d'existence des solutions. Rev. Roum. Math. Pures Appl. 20, 631-657,1975.

[6] E, Campu ; Approximation des solutions des équations différentielles au paratingent à retardement, dans les espaces de Banach. Rev. Roum. Math. Pures Appl. 25, 509-518,1980.

[7] C. Castaing, A. G. Ibrahim; Functional differential inclusions on closed sets in Banach spaces. Adv. Math. Eco. 2, 21-39, 2000.

[8] K. Deimling; Multivalued differential equations, De Gruyter Ser. Nonlinear Anal. Appl. 1, Walter de Gruyter, Berlin, New York, 1992.

[9] H.Frankowska, F.Rampazzo ; Filippov's and Filippov-Wasewski theorems on closed domains. J. Diff. Equ, 161, 449-478, 2000

[10] G. Haddad; Monotone trajectories of differential inclusions and functional differential inclusions with memory, Israel J. Math. 39(1-2), 83-100. 1982.

[11] G. Haddad; Monotone viable trajectories for functional differential inclusions with memory. J. Diff. Eq. 42, 1-24, 1981.

[12] G. Haddad; Functional viability theorems for functional differential inclusions with memory. Ann. Inst. Henri Poincaré I (3), 179-204, 1984.

[13] G. Haddad, J. M. Lasry; Periodic solutions of functional differential inclusions and fixed points of $\sigma$-selectionable correspondences. J. Math. Anal. Appl. 96, 295-312, 1993.

[14] M. Hukuhara; Sur l'application semi-continue dont la valeur est un compact convexe. RIMS-11, Res. Inst. Math. Sci. Kyoto. Univ. 941-945, 1963.

[15] M. Kamenskii, V.Obukhovskii, P. Zecca; Condensing multivalued maps and semilinear differential inclusions in Banach spaces, De Gruyter Ser. Nonlinear Anal. Appl. 7, Walter de Gruyter, Berlin, New York, 2001.

[16] M. Kisielewicz; Differential Inclusions and Optimal Control. Kluwer, Dordrecht, The Netherlands, 1991.

[17] B. Kryzowa; Equation au paratingent à argument retardé. Ann. Univ. Marie Curie-Sklodwska. Sectio A, 17, 7-18, 1965. 
[18] B. Kryzowa; Sur les familles de solutions des équation au paratingent à argument retardé. Ann. Univ. Marie Curie-Sklodwska. Sectio A, 17, 19-24, 1965.

[19] A. D. Myshkis; General theory of differential equations with delayed argument, Uspehi Matem. Nauk 4, no. 5, 1949, 99-141 (in Russian)

[20] S. Raczynski; Differential inclusions in system simulation. Trans. Society for Computer Simulation, Vol. 13. No. 1, 47-54. 1996.

[21] S. Raczynski; A market model: uncertainty and reachable sets. Int. J. Simul. Multisci. Des. Optim. 6, A2, 2015.

[22] S. Raczynski; Creating galaxies on a PC. Simulation, Vol 74, No. 3, 161-166, 2000.

[23] G. V. Smirnov; Introduction to the theory of differential inclusions, Amer. Math. Soc., Providence, R.I. 2002.

[24] E.D Sontag; An infinite-time relaxation theorem for differential inclusions. in Proc. Amer. Math. Soc., V 131, N² 2, 487-499, 2001.

[25] E.D Sontag ; A relaxation theorem for differential inclusions with applications to stability properties. in Mathematical Theory of Networks and Systems (D. Gilliam and J. Rosenthal, eds.), 12 pages, August 2002, Electronic Proceedings of MTNS-2002

[26] A. Syam; Contribution à l'etude des inclusion différentielles. Doctorat Thesis, Université Montpellier II, 1993.

[27] A. Turowicz; Sur les trajectoires et les quasitrajectoires des systèmes de commande nonlinéaires. Bull. Acad. Polon. Ser. Sci. Math., Astr., Phys, Vol 10, 529-531, 1963.

[28] T. Wasewski; Sur une genéralisation de la notion des solutions d'une équation au contingent. Bull. Acad. Pol. Sci. Ser. Math. Astronom. Physi. 10 No. 1. Warszawa, 1962.

[29] T. Wasewski; Sur les systèmes de commande non lineaires dont le contredomaine n'est pas forcement convexe. Bull. Acad. Pol. Sci. Ser. Math. Astronom. Physi. 10 No. 1. Warswa. 1962.

[30] T.Wazewski; Sur un système de commande dont les trajectoires coüncident avec les quasitrajectoires d'un système de commande donné. Bull. Acad. Pol. Sci. Ser. Math., Astronom., Physi. 11, N 3. Warszawa 1963.

[31] W. Zygmunt; On a certain pratingent equation with a deviated argument. Ann. Univ. Marie. Curie-Sklodwska. Lublin. Polonia. 18, 14, 127-135, 1974.

[32] W. Zygmunt; On some properties of a certain family of solutions of the paratingent equation. Ann. Univ. Marie Curie-Sklodowska, Lublin. Polonia. Sect. A -28, 136-141,1976. 